\title{
Management of calcaneal fractures: a case series
}

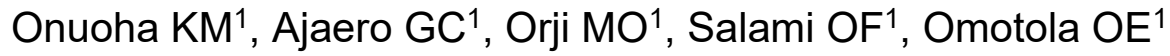 \\ ${ }^{1}$ Deprtment of Surgery, Babcock University Teaching Hospital, Ilishan-Remo, Ogun State, Nigeria
}

Submitted: $3^{\text {rd }}$ June 2020

Accepted: $10^{\text {th }}$ August 2020

Published: $30^{\text {th }}$ December 2020

\begin{abstract}
Background

Untreated or inappropriately treated, calcaneal fractures can result in arthritis, chronic ankle pain, and ankle deformity which can significantly impact the lifestyle of affected patients. Even though calcaneal fractures are common among those with multiple injuries, they can easily be missed, and not treating them would significantly impact on the patient negatively. It is therefore important a high index of suspicion is made for this injury among patients involved in high-energy trauma.

Case Presentation

This article is a retrospective recall of the six patients seen with calcaneal fractures that were treated in our hospital between 2010 and 2018, they were mainly multiply injured and the advanced trauma life support (ATLS) protocol helped to delineate these fractures, the use of modern-day operative and non-operative methods of management and rehabilitation were employed.

Discussion and conclusion

Calcaneal fractures are mostly associated with high-energy trauma which includes falls from heights or vehicle accidents with almost $50 \%$ soft tissue involvement. This is similar to cases presented in this paper as all the patients were either involved in road traffic accidents or had fallen from a height, however, soft tissue destruction manifesting as open fractures were absent in our series. Calcaneal fractures are rare and can easily be missed as patients may present as multiply injured. Prompt and adequate Rehabilitation is important to improve function.
\end{abstract}

Keywords: Calcaneal fracture, Intra-articular fractures, Heel bone fractures, Tarsal bone injuries, Trauma.

\section{Background}

Calcaneal fractures are rare and account for about $1-2 \%$ of all fractures occurring in the human body and $60 \%$ of all tarsal bones' injuries (1). It is mostly associated with high energy axial load applied to the heel which drives the talus downward onto the calcaneus. Such trauma includes falls from a height or head-on collision motor vehicle accidents. Due to the high energy of the trauma, soft tissues are commonly involved with $50 \%$ presenting with the combined lesion (2). Calcaneal fractures can be intra-articular or extraarticular. Intra-articular fractures account for approximately $70 \%$ of calcaneal fractures and are more challenging to manage with the worse outcomes. The Calcaneus, also called the heel bone, is a large bone that forms the foundation of the rear part of the foot (3).

Patients that sustain calcaneal fractures commonly present with pain over the heel, inability to weight bear on the affected foot with limited mobility, and an antalgic gait. There may be swelling, redness, and hematoma over the area. Mondor's Sign, which occurs when the hematoma extends to the sole and described as pathognomonic for calcaneal fractures (4). The heel may become edematous and widened as a result of the displacement of the calcaneal border. The soft tissues must be evaluated as injury to the soft tissue has been associated with significant complications $(5,6)$. During

Correspondence: Onuoha, Kenechukwu M.

Department of Surgery, Babcock University Teaching Hospital, Ilishan, Nigeria

+2348034128159; mckelng@yahoo.com

(c) BUMJ. 2020 Open Access This article is distributed under the terms of the Creative Commons Attribution 4.0 International License 
examination, passive extension of the toes should be performed to assess for an unexpected pain in the deep arch of the foot. Skin wrinkling with dorsiflexion and eversion of the hindfoot is a rough guide to the acceptable level of swelling to allow for an extensive approach. This should, therefore, be assessed before considering surgical intervention (7). The advanced trauma life support protocol is a very useful approach not to miss out on calcaneal fractures which may be missed in the case of multiply injured patients. The rehabilitation of such patients is also very important to get good functional outcomes.

\section{Case reports}

\section{Case one}

A 54-year old man involved in a road traffic accident, he was a restrained driver and was hit

Figure 1: X-ray of the right knee Joint (Lateral view) showing a patella fracture

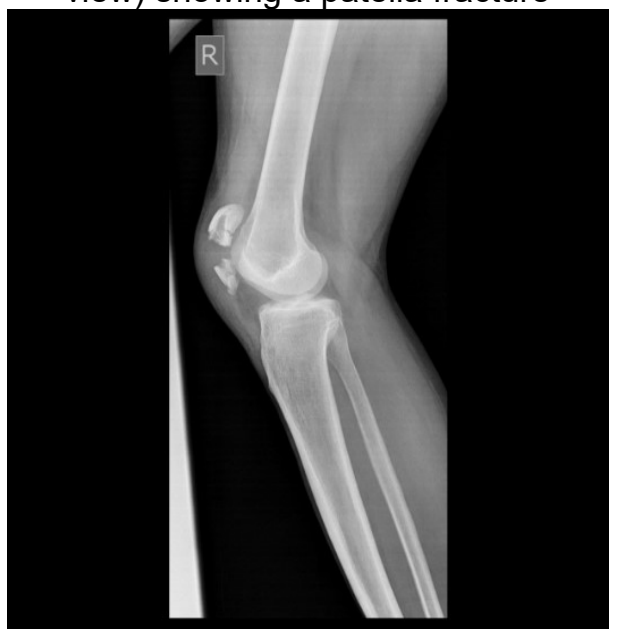

His patella was repaired with tension band wiring of the transverse fracture. His closed calcaneal fracture was treated via a closed reduction and percutaneous Kirschner wire fixation (Figures 3 and 4). He was discharged five days after his surgery on non-weight bearing with bilateral axillary crutches. He was followed up at our outpatient clinic. He had an uneventful recovery which was evident in his seventh month. He was seen at the outpatient clinic and was noted to have a union and was ambulating well without support.

\section{Case Two}

A 42-year-old male victim of a road traffic accident presented to the accident and emergency unit with multiple injuries four days by another car from the front via a head-on collision. He was taken to a nearby hospital for immediate management and was referred to us the next day for expert care. On examination, there was tenderness of the left hip, swollen right knee with a reduced range of motion. His right ankle was swollen, with a reduced range of motion and he was unable to weight-bear on his right lower limb. A radiograph of the knee showed a displaced transverse patellar fracture and his ankle radiograph showed an oblique extraarticular fracture of the calcaneus. There was a disruption of the superior and inferior cortices, normal articular margins with the ankle mortise still intact. There was no significant displacement, however, soft tissue swelling was noted. These are shown in Figures 1 and 2 respectively.

Figure2: X-ray of the right foot (Lateral view) showing a calcaneal fracture

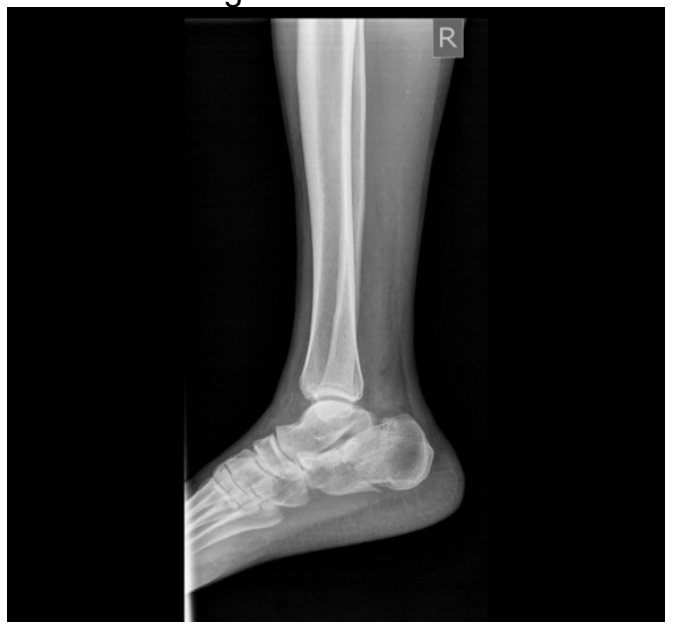

after his accident. He was involved in a head-on collision with a fast-moving vehicle when he fell asleep on the wheel. He was taken to the local hospital where he regained consciousness.

His injuries included; facial abrasions closed injury to the left arm and left foot, with associated laceration of the left knee. Radiographs showed a left humeral shaft fracture, comminuted supracondylar fracture of the femur, fracture of the left medial malleolus, and a talar fracture. Ankle x-ray showed right comminuted, minimally displaced intra-articular calcaneal fracture.

$\mathrm{He}$ underwent open reduction and internal fixation of the left talus, debridement of the left knee injury, and closed reduction with Kirschner wiring of the right calcaneus. After four days, the patient had closed reduction and internal fixation 
with an interlocking nail of the left humerus and retrograde femoral nailing of his left femur. He was discharged home 10 days after the first

Figure 3: X-ray of the right foot, (AP) view showing Kirschner wires used for ORIF

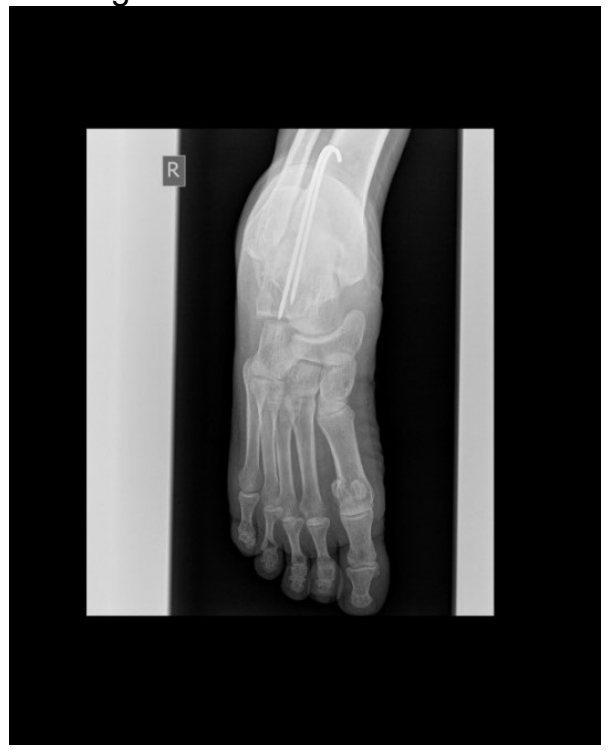

\section{Case Three}

A 42-year old lady who presented after accidentally falling into a deep ditch and could no longer bear weight on the left foot, on examination she had a left swollen ankle with deformity (valgus deformity), tenderness and reduced range of motion of the left foot. Her anterior and lateral ankle radiograph showed an oblique calcaneal fracture with disruption of the plantar cortex and subtle reduction of calcaneal

Figure 5: Lateral view x-ray of the left foot showing extra-articular oblique fracture

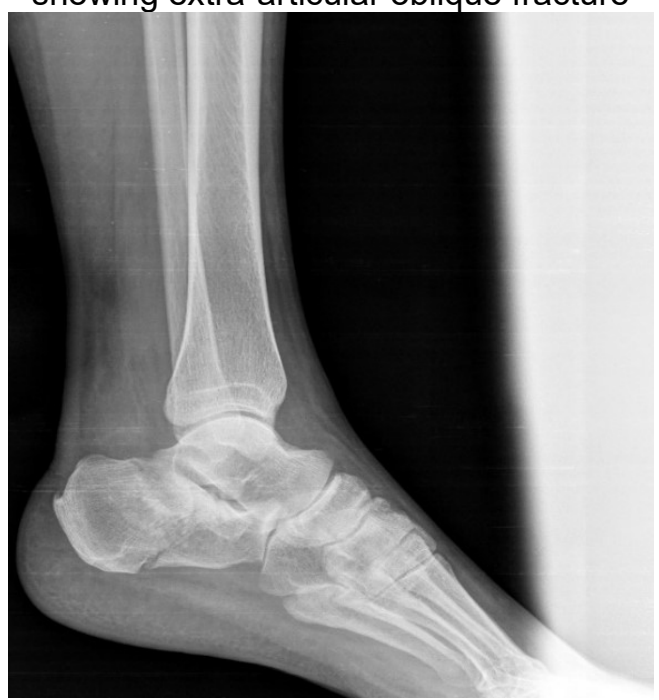

surgery with a below-knee cast after training on wheelchair ambulation.

Figure 4: Lateral view X-ray of the right knee showing tension band wiring for patella fracture

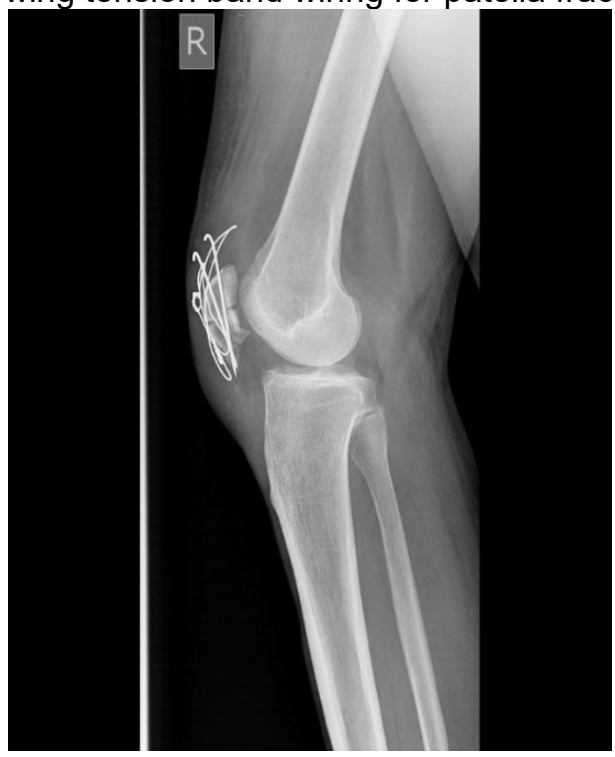

height as shown in Figure 5. Periarticular soft tissue swelling was also noted. She subsequently had a percutaneous cannulated screw fixation (figure 6). She was discharged the next day with bilateral axillary crutches.

Four months after discharge, she was noted to still have intermittent foot pain and swelling, however by the end of one year, the swelling had completely resolved as well as the foot pain and she was fully ambulating.

Figure 6: Lateral view X-ray of the foot showing cannulated screw fixation of the calcaneus

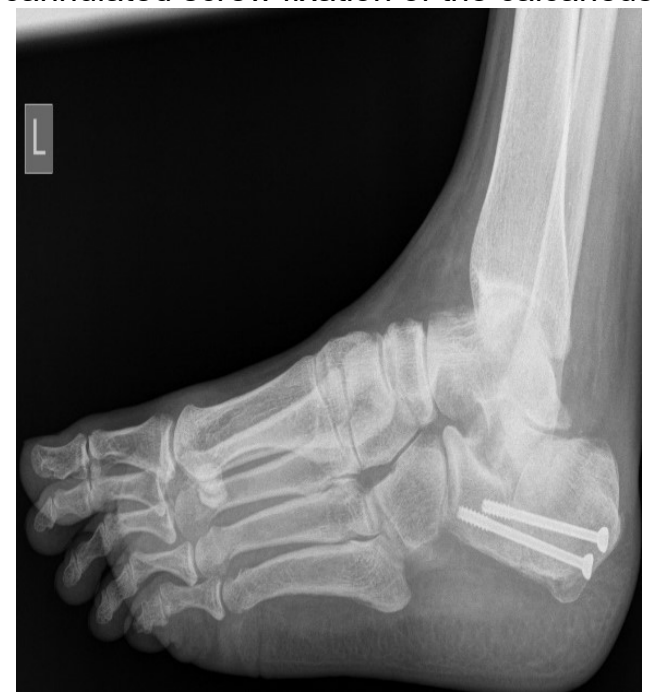




\section{Case four}

A 56-year old male was presented with inability to bear weight on his left lower limb. He fell from a height and landed on his left foot. On examination, the left foot was swollen and tender with ankle x-ray showing a left extra-articular displaced fracture. He underwent a closed reduction with $\mathrm{K}$-wire fixation of the calcaneus.

The patient was discharged home the following day with pain relief and an air cast boot. Ambulation with bilateral axillaries crutches and avoidance of weight-bearing on the left lower limb was advised. Four weeks' post-operative review showed that he was stable on crutches with satisfactory healing.

\section{Case five}

A 36year old male presented with an inability to bear weight on both lower limbs. He fell from a 2meter height and landed in an awkward position. On examination, both ankles were tender and swollen with a range of motion, no open wound, and no injury on any other part of his body.

Ankle radiograph showed an intra-articular calcaneal fracture on the right and extra-articular calcaneal fracture on the left ankle. He underwent percutaneous reduction and Kirschner wire fixation of both left and right calcanei.

Post-surgery, the patient's limbs were elevated; he had bilateral below knee back slab and was discharged seven days after his surgery with a wheelchair for mobility. He was trained on wheelchair transfer from the bed and vice versa.

\section{Case six}

A 30-year old man presented 6 hours after jumping a fence with the inability to move both lower limbs. He had severe pain on both limbs with no abrasion, bleeding, and no other injury of note. On examination, both ankles were swollen, warm to touch, and tender. His ankle $x$-ray showed a displaced intra-articular fracture of the calcaneus. His surgical management involved closed reduction and K-wire fixation of the left calcaneus.

He was discharged three days after surgery with bilateral axillary crutches and advised on nonweight bearing on the left with partial weightbearing on the right lower limb. He also had outpatient physiotherapy sessions.

At the 3rd month post-operative review, the patient was mobilizing with both limbs and only occasional pain.

\section{Discussion}

The low incidence $(1-2 \%)$ of calcaneal fractures was reflected in this case series with only six occurring in this orthopedic center within 8 years. The multiply injured patients managed using the advanced trauma life support also helped to identify these cases which could have easily been missed. This case series reflects the rarity of the fracture with six patients, however, one had bilateral fractures making a total of seven calcaneal fractures. Four were intra-articular and three were extra-articular fractures which reflects the more cases of intra-articular fractures documented in literature (8).

As regards gender, males are usually more affected as they are more active in the population (9) and this was the observation in our review. Same may be obtained for the reason why we had the young and middle-aged affected in this series (10).

The documented mechanism of injury is also seen in our series, furthermore, none of the cases was an open fracture which precludes soft tissue healing, and this is similar to documented evidence in the literature, as most of the cases seen are closed fractures (11).

The typical "Lovers fracture" which is a calcaneal fracture sustained following a jump from a height is also well documented (12) and is seen in some of our cases. However, the term 'lovers fracture' is not used again as the circumstances leading to the terminology is not frequent.

The decision to manage conservatively or not depends on the nature of the injury, the expertise of the surgeon, the availability of equipment, and the finance of the patient (13). Generally, conservative treatment is offered to those with minimal injury and non-displaced fractures which are extra-articular. This is done with the use of boot casts or air-cast boots as was done for our patients (14).

Kirschner wires were used for the fixation as they are fine and non-traumatic and therefore ideal for the cancellous nature of the calcaneus (15) these were easily removed at the outpatient when the fracture healed. The choice of cannulated lag screws was also done for the extra-articular fracture which required ORIF and no plan for later removal. Thus a decision has to be made before surgery if the implants to be used will be removed or not which will impact the nature of surgery to be carried out (16). The use of the intra-operative imaging at surgery aids close reduction and internal fixation thus reducing the injury to soft tissues with poor healing potential (17). 
Healing of calcaneal fractures could be very precarious as the blood supply is poor and the bone is mainly cancellous in nature (18), hence, strict non-weight bearing must be done in the early phases of treatment and also attention to wound care mandatory to prevent infection or wound breakdown (19).

Known complications of calcaneal fractures include osteomyelitis, non-union, and wound breakdown (20) and these were not seen in our patients even at long term follow up. Rehabilitation is a continuous process and may take up to 6 months (21) walking aids like air-cast boots, axillary crutches are commonly used.

\section{Conclusion}

Calcaneal fractures are rare and can easily be missed as patients may present as multiply injured. Conservative or operative treatments give good outcomes depending on the nature of the fracture. Rehabilitation is important to improve function. Our experience with these cases may serve as a guide for proper management.

Improvement in radiological assessment and the use of intra-operative imaging ensures that the fixation is adequate. Rehabilitation using air-cast boots for ambulation is a current trend in modernday orthopedics which has led to improved management of this fracture.

However, a high index suspicion should be applied for patients presenting with high energy trauma with or without multiple injuries.

\section{Declarations}

Ethics approval and consent to participate

The authors obtained consent and permission to publish the data and images from the concerned patients.

\section{Consent for publication}

The authors hereby give consent for the publication of our work under the creative commons CC Attribution-Noncommercial 4.0 license

\section{Availability of data and materials \\ Not applicable}

\section{Competing interests}

The authors have declared no conflict of interest.

\section{Funding}

The authors received no research funding.

\section{Authors' contributions}

AGC conceptualized the study, while OKM performed patient follow-up, image collation, and wrote the first draft of the manuscript. OMO conducted the literature review and SOF oversaw the data collection process. OOE contributed to literature review. All the authors read and approved the final draft.

Acknowledgment

We acknowledge the patients who offered themselves, and also the theatre staff who cooperated with the team to Publish this article.

\section{References}

1. Meng $Q$, Wang $Q$, Wu X, Peng A, Yan J. Clinical application of the sinus tarsi approach in the treatment of intra-articular calcaneal fracture. Medicine. 2018 Mar;97(13). https://doi.org/10.1097/md.00000000000101 $\underline{75}$

2. Leite CB, Macedo RS, Saito GH, Sakaki MH, Kojima KE, Fernandes TD. Epidemiological study on calcaneus fractures in a tertiary hospital. Revista Brasileira de Ortopedia. 2018 Aug;53(4):472-6. https://doi.org/10.1016/i.rboe.2018.05.014

3. Heel Pain and Heel Fracture Specialist. Foot and Ankle Specialists. Available at https://www.nefootdocs.com/heel-pain-heelfractures.html

4. Richman JD, Barre PS. The plantar ecchymosis sign in fractures of the calcaneus. Clinical Orthopaedics and Related Research. 1986 Jun 1;207:122-5. https://doi.org/10.1097/00003086198606000-00022

5. Berry GK, Stevens DG, Kreder HJ, McKee M, Schemitsch E, Stephen DJ. Open fractures of the calcaneus: a review of treatment and outcome. Journal of orthopaedic trauma. 2004 Apr $1 ; 18(4): 202-6$. https://doi.org/10.1097/00005131200404000-00002

6. Heier KA, Infante AF, Walling AK, Sanders RW. Open fractures of the calcaneus: softtissue injury determines outcome. JBJS. 2003 Dec $1 ; 85(12): 2276-82$. https://doi.org/10.2106/00004623200312000-00002

7. Forsthoefel C. Calcaneal-fracture. Available at

https://www.orthobullets.com/trauma/1051/ca Icaneus-fractures Updated: 6/14/2020

8. Sanders R. Current concepts reviewdisplaced intra-articular fractures of the calcaneus. JBJS. 2000 Feb 1;82(2):225-50. 
https://doi.org/10.2106/00004623200002000-00009

9. Leite CB, Macedo RS, Saito GH, Sakaki MH, Kojima KE, Fernandes TD. Epidemiological study on calcaneus fractures in a tertiary hospital. Revista Brasileira de Ortopedia. 2018 Aug;53(4):472-6. https://doi.org/10.1016/i.rboe.2018.05.014

10. Khetan VV, Patel I, Modi DR, Panchal N, Bhavsar N, Joshi A. Functional outcome of calcaneal fractures treated by various methods. Nigerian Journal of Orthopaedics and Trauma. 2019 Jul 1;18(2):44. https://doi.org/10.4103/njot.njot $8 \quad 19$

11. Cross III WW, Swiontkowski MF. Treatment principles in the management of open fractures. Indian journal of orthopaedics. 2008 Oct;42(4):377. https://doi.org/10.4103/00195413.43373

12.Davis D, Seaman TJ, Newton EJ. Calcaneus Fractures. InStatPearls [Internet] 2020 Apr 24. StatPearls Publishing.

13. Rammelt S, Sangeorzan BJ, Swords MP. Calcaneal fractures-should we or should we not operate?. Indian Journal of Orthopaedics. 2018 Jun;52:220-30. https://doi.org/10.4103/ortho.ijortho 55517

14. Türkmen F, HakkÄ \pm Korucu I, Sever C, Göncü G, KaçÄ \pm ra BK, Toker S. Calcaneal fractures, treatments and problems. Clinical Research on Foot \& Ankle. 2014 Apr 5:1-6.

15. Wu J, Zhou F, Yang L, Tan J. Percutaneous reduction and fixation with Kirschner wires versus open reduction internal fixation for the management of calcaneal fractures: a metaanalysis. Scientific reports. 2016 Jul 26;6:30480.

https://doi.org/10.1038/srep30480

16. Reith G, Schmitz-Greven V, Hensel KO, Schneider MM, Tinschmann T, Bouillon B, Probst C. Metal implant removal: benefits and drawbacks-a patient survey. BMC surgery. 2015 Dec 1;15(1):96. https://doi.org/10.1186/s12893-015-0081-6

17. Walde TA, Sauer B, Degreif J, Walde HJ. Closed reduction and percutaneus Kirschner wire fixation for the treatment of dislocated calcaneal fractures: surgical technique, complications, clinical and radiological results after $2-10$ years. Archives of orthopaedic and trauma surgery. 2008 Jun 1;128(6):585-91. https://doi.org/10.1007/s00402-008-0590-1

18. Attinger C, Cooper P. Soft tissue reconstruction for calcaneal fractures or osteomyelitis. Orthopedic Clinics. 2001 Jan 1;32(1):135-70.

https://doi.org/10.1016/s00305898(05)70199-1

19. Backes M, Schepers T, Beerekamp MS, Luitse JS, Goslings JC, Schep NW. Wound infections following open reduction and internal fixation of calcaneal fractures with an extended lateral approach. International orthopaedics. 2014 Apr 1;38(4):767-73. https://doi.org/10.1007/s00264-013-2181-1

20.Kim Y, Inori F, Yamanaka K, Murakami S, Narita E, Yamamura K, Yasuda H, Fukuda M, Konishi S, Minoda Y. A Case of Osteomyelitis after Calcaneal Fracture Treated by AntibioticContaining Calcium Phosphate Cements. Case Reports in Orthopedics. 2018 Jun $12 ; 2018$. https://doi.org/10.1155/2018/9321830

21. Schmal H, Larsen AH, Froberg L, Erichsen JL, Madsen CF, Pedersen L. The effect of a heelunloading orthosis in short-term treatment of calcaneus fractures on physical function, quality of life and return to work-study protocol for a randomized controlled trial. Trials. $2019 \quad$ Dec;20(1):1-8. https://doi.org/10.1186/s13063-019-3447-8 